\title{
A Third-Order Consensus Approach for Vehicle Platoon with Intervehicle Communication
}

\author{
Jianzhong Chen $(\mathbb{D}$, Dongyang Bai $\mathbb{D}$, Huan Liang $\mathbb{D}$, and Yang Zhou $(\mathbb{D})$ \\ School of Automation, Northwestern Polytechnical University, Xian 710072, China \\ Correspondence should be addressed to Jianzhong Chen; jzhchen@nwpu.edu.cn
}

Received 18 May 2018; Accepted 9 July 2018; Published 24 July 2018

Academic Editor: Giulio E. Cantarella

Copyright (C) 2018 Jianzhong Chen et al. This is an open access article distributed under the Creative Commons Attribution License, which permits unrestricted use, distribution, and reproduction in any medium, provided the original work is properly cited.

A third-order consensus approach is proposed for the vehicle platoon. For addressing the platoon problem, a realistic, third-order vehicle dynamics model is used, and the spacing policy and the vehicle acceleration error are embedded into the consensus protocol. A sufficient and necessary condition of asymptotically stability is obtained for the vehicle platooning system. Numerical simulations for several traffic scenarios are carried out. The results demonstrate the effectiveness and the robustness of the presented approach.

\section{Introduction}

Traffic congestion is a serious problem and considerable challenge in many parts of the world. How to alleviate traffic congestion has attracted great concern in recent years. Platoon based cooperative driving is one of the promising approaches to improve traffic flow, enhance traffic capacity, and reduce fuel consumption (see [1-3] and the references therein). The main goal of vehicle platoon control is to ensure that all vehicles keep the consensus speed and maintain the desired intervehicle distance prespecified by the spacing policy. The platoon studies can date back to the Partners for Advanced Transportation Technology (PATH) program [4]. Since then, researchers have introduced and implemented various control strategies such as the consensus control, model predictive control [5], optimal control [6], sliding-mode control [7], and $H_{\infty}$ control [8].

This paper is concerned with the consensus control strategy for platooning of vehicles. Consensus control is an active research field in multivehicle cooperative control. The pioneering work has been reported by Fax and Murray [9]. They have developed a theoretical framework of consensus for cooperative control of multiple vehicles. They focus on vehicles with first order dynamics and consider fixed time delays and different communication topologies. Later, Ren [10] has studied cooperative control of vehicles modeled by secondorder dynamics and introduced consensus strategies under directed information topologies. Wang et al. [11] have proposed a weighted and constrained consensus control strategy for platoon coordination. They have studied the consensus control under a stochastic framework. The communication noises are considered while time-varying delays are not taken into account.

di Bernardo et al. [12] have investigated the vehicle platooning problem in the presence of heterogeneous timevarying delays, introduced a distributed control protocol to guarantee second-order consensus in vehicles platoon, and proved the stability of platoon based on LyapunovRazumikhin theorem. di Bernardo et al. [13] have modified the spacing policy in the control strategy and extended the approach in [12]. The proposed algorithm is validated by experiments performed on a three-vehicle platoon. Santini et al. $[14,15]$ have also proposed a second-order consensus algorithm for the vehicle platoon with intervehicle communications. The constant time headway $(\mathrm{CTH})$ spacing policy and the time-varying delays are embedded in the algorithm. The performance of the algorithm is compared with a well-known Cooperative Adaptive Cruise Control (CACC) algorithm and is validated in the realistic scenario. In [16], the platoon problem in the presence of malicious attacks is studied, and a new second-order consensus strategy has been proposed to enhance the protection level of platoons. The designed strategy is validated by analytical and experimental results. Yan et al. [17] have presented a control strategy for 
vehicle platoon to deal with the actuator saturation and absent velocity measurement.

In $[18,19]$, the authors have suggested a distributed control strategy to achieve third-order consensus of a dynamic network in the presence of time-varying heterogeneous delays. Saeednia and Menendez [20] have discussed the truck platooning problem and presented a distributed algorithm based on the average consensus algorithm. They have compared the distributed algorithm with a centralized optimization-based algorithm by simulating multiple scenarios. Wang et al. [21] have suggested the distributed consensus algorithm and protocol for CACC system. Zegers et al. [22] have adopted a realistic longitudinal vehicle dynamics model and the CTH spacing policy for the consensus problem. A three-vehicle platoon test is used to validate the performance of the control approach.

Jia and Ngoduy [23] have considered the packet loss and transmission delay and developed consensus control algorithms for the multiple platoons cooperative driving. Jia and Ngoduy [24] have further studied the cooperative model considering vehicle-to-vehicle (V2V) and vehicleto-infrastructure (V2I) communication and suggested an improved consensus control strategy. In [25], the authors have suggested a control algorithm of mixed vehicle platoon based on a unified model, in which the connected and autonomous vehicle and the human-driven vehicle are described by the different control models.

In this paper, we propose a novel third-order consensus strategy for the vehicle platooning system. Comparing with the studies $[18,19]$, the paper has two contributions. Firstly, the leader's acceleration is not assumed to be zero in our consensus algorithm, which is more adaptable to complex driving environments. Secondly, the speed errors between following vehicles are taken into account, whereas this factor has not been considered in the works $[18,19]$. In the cooperative driving environment, the introduction of the speed error information can further improve the stability of traffic flow [26-29]. The asymptotic platoon stability is investigated by using Lyapunov-Razumikhin theorem. The effectiveness of the proposed approach is evaluated by simulations for several traffic scenarios.

The rest of the paper is organized as follows. In Section 2, the mathematical preliminaries are introduced. In Section 3, we present a third-order consensus control algorithm and carry out the stability analysis. Numerical simulations can be found in Section 4. Some conclusions are drawn in Section 5.

\section{Mathematical Preliminaries}

Suppose that the platoon consists of a leader vehicle (labeled with 0 ) and $N$ following vehicles. The intervehicle communication structure of the followers is described by a directed graph (digraph) $G=(V, E, A)$ in which $V=\{1,2, \cdots, N\}$ is the set of nodes, $E \subseteq V \times V$ denotes the set of edges, and $A=\left[\alpha_{i j}\right]_{N \times N}$ represents the adjacency matrix. In this paper, we assume $\alpha_{i j}=1$ if vehicle $i$ can receive the information from vehicle $j$; otherwise, $\alpha_{i j}=0$. Moreover, we assume that there are no self-loops in the digraph; i.e., $\alpha_{i i}=0$. The degree matrix
$D=\operatorname{diag}\left\{d_{1}, \cdots, d_{n}\right\}$ is diagonal matrix, whose elements are $d_{i}=\sum_{j=1}^{N} \alpha_{i j}$. The Laplacian matrix of the directed graph $L$ is defined as $L=D-A$. We also consider another graph $\bar{G}$ to model the information exchange among $N$ followers and the leader. To investigate the leader-following problem, we define a diagonal matrix $B=\operatorname{diag}\left\{b_{1}, \cdots, b_{n}\right\}$ to be a leader adjacency matrix associated with the platoon consisting of $N$ following vehicles and one leader (labeled with 0 ), where $b_{i}>0$ if node 0 is a neighbor of node $i$; otherwise, $b_{i}=0$. We suppose that node 0 is globally reachable in $\bar{G}$, which means there is a path in $\bar{G}$ from every node $i$ in $G$ to node 0 [30].

We next recall some important lemmas and theorems used in studying the stability of the vehicle platoon system.

Let $C\left([-r, 0], R^{n}\right)$ be a Banach space of continuous functions from $[-r, 0]$ into $R^{n}$ with a norm $\|\phi\|_{c}=$ $\max _{\theta \in[-r, 0]}\|\phi(\theta)\|$, where $\|\cdot\|$ is Euclidean norm. Consider the following time-delay system:

$$
\begin{aligned}
& \dot{x}(t)=f\left(t, x_{t}\right), \quad t>0, \\
& x(\theta)=\phi(\theta), \quad \theta \in[-r, 0],
\end{aligned}
$$

where $x_{t}(\theta)=x(t+\theta), \forall \theta \in[-r, 0], f: R \times C\left([-r, 0], R^{n}\right) \longrightarrow$ $R^{n}$ is a continuous function, and $f(t, 0)=0, \forall t \in R$. Then the following result holds.

Theorem 1 (Lyapunov-Razumikhin theorem, [31]). Let $\varphi_{1}$, $\varphi_{2}$, and $\varphi_{3}$ be continuous, nonnegative, nondecreasing functions with $\varphi_{1}(s)>0, \varphi_{2}(s)>0$, and $\varphi_{3}(s)>0$ for $s>0$ and $\varphi_{1}(s)=\varphi_{2}(s)=0$. If there is a continuous function $V(t, x)$ such that

$$
\varphi_{1}(\|x\|) \leq V(t, x) \leq \varphi_{2}(\|x\|), \quad t \in R, x \in R^{n},
$$

if, in addition, there exists a continuous nondecreasing function $\varphi_{4}(s)$ with $\varphi_{4}(s)>s, s>0$ such that

$$
\dot{V}(t, x) \leq-\varphi_{3}(\|x\|),
$$

and if $V(t+\theta, x(t+\theta)) \leq \varphi_{4}(V(t, x(t))), \theta \in[-r, 0]$, then the solution $x=0$ is uniformly asymptotically stable.

Lemma 2 (Hermite-Hadamard inequality, [32]). Let $f:[a$, $b] \longrightarrow R$ be a convex function; then

$$
f\left(\frac{a+b}{2}\right) \leq \frac{1}{b-a} \int_{a}^{b} f(x) d x \leq \frac{f(a)+f(b)}{2} .
$$

Lemma 3 (see [30]). For any $c, d \in R^{n}$ and any positivedefinite matrix $E \in R^{n \times n}$, it holds that

$$
2 c^{T} d \leq c^{T} E c+d^{T} E^{-1} d .
$$

\section{Platooning Control}

The cooperative driving strategy of the platoon is to make each member of the platoon follow the leader's behavior and maintain the desired small intervehicle spacing. Consider a platoon consisting of $N$ following vehicles and a leader 
moving along a single lane. The ith vehicle's longitudinal dynamics can be described [33]:

$$
\begin{aligned}
\dot{x}_{i}(t) & =v_{i}(t), \\
\dot{v}_{i}(t) & =a_{i}(t), \\
\dot{a}_{i}(t) & =\frac{1}{T_{i}}\left[u_{i}(t)-a_{i}(t)\right],
\end{aligned}
$$

where $x_{i}, v_{i}$, and $a_{i}$ are, respectively, the position, speed, and acceleration of the $i$ th vehicle, $u_{i}$ denotes the desired acceleration which is the control input, and $T_{i}$ is the time constant of the drivetrain.

The consensus control goal of the platoon can be expressed as

$$
\begin{aligned}
& x_{i}(t) \longrightarrow x_{0}(t)-i \cdot s, \\
& v_{i}(t) \longrightarrow v_{0}(t), \\
& a_{i}(t) \longrightarrow a_{0}(t),
\end{aligned}
$$

where $s$ is the desired distance between two adjacent vehicles which can be set according to a constant spacing policy studied in [34], and $i \cdot s$ is the desired distance of vehicle $i$ from the leader 0.

3.1. Consensus Control Algorithm. To achieve the control goal that the platoon members follow the leader's state, we design the following consensus control algorithm embedding the spacing policy information and the time-varying communication delays:

$$
\begin{aligned}
& u_{i}(t)=\sum_{j=1}^{N} \alpha_{i j} \beta_{1}\left[x_{j}\left(t-\tau_{j}\right)-x_{i}(t)-(i-j) \cdot s\right. \\
& \left.\quad+v_{0}\left(t-\tau_{0}\right) \tau_{j}\right] \\
& +\sum_{j=1}^{N} \alpha_{i j} \beta_{2}\left[v_{j}\left(t-\tau_{j}\right)-v_{i}(t)\right] \\
& +b_{i}\left\{\beta_{1}\left[x_{0}\left(t-\tau_{0}\right)-x_{i}(t)+v_{0}\left(t-\tau_{0}\right) \tau_{0}-i \cdot s\right]\right. \\
& +\beta_{2}\left[v_{0}\left(t-\tau_{0}\right)-v_{i}(t)\right] \\
& \left.+\beta_{3}\left[a_{0}\left(t-\tau_{0}\right)-a_{i}(t)\right]\right\} \\
& +a_{0}\left(t-\tau_{0}\right),
\end{aligned}
$$

where $\beta_{1}>0, \beta_{2}>0$, and $\beta_{3}>0$ are the control parameters; $(i-j) \cdot s$ is the desired spacing errors between vehicles $i$ and $j$ which is set according to the spacing policy; and $\tau_{0}$ and $\tau_{j}$ are, respectively, the time-varying communication delays from the leader and from the vehicle $j$ to the vehicle $i$. Here, the effect of position difference is ignored and it is assumed that all neighboring vehicles can receive the beacon simultaneously from the leader and the vehicle $j$.

The algorithm (8a), (8b), (8c), (8d), (8e), and (8f) can be described in detail as follows:
(1) (8a) is the position error between the distance of vehicle $i$ and vehicle $j$ with respect to the desired distance $(i-j) \cdot s$. The term $v_{0}\left(t-\tau_{0}\right) \tau_{j}$ is introduced as the distance compensation due to the time-delay $\tau_{j}$ of $x_{j}$.

(2) (8b) represents the velocity error between members $i$ and $j$.

(3) (8c) represent the position error between the distance of vehicle $i$ and the platoon leader 0 with respect to the desired distance $i \cdot s$. The term $v_{0}\left(t-\tau_{0}\right) \tau_{0}$ is added as the distance compensation due to the time-delay $\tau_{0}$ of $x_{0}$.

(4) $(8 d)$ and (8e) represent, respectively, the velocity error and acceleration error between member $i$ and the platoon leader 0 . The leader acceleration is introduced in (8f).

According to (8a), (8b), (8c), (8d), (8e), and (8f), the consensus algorithm is designed based on state errors between the vehicle itself and the delayed state information of its neighboring vehicles obtained via wireless communication. The acceleration error is embedded into the proposed algorithm. The control algorithm using acceleration information has some advantages such as improving control reactivity and avoiding vehicle falling too far behind the vehicle ahead [19]. The leader's acceleration is not assumed to be zero in the algorithm (8a), (8b), (8c), (8d), (8e), and (8f) to adapt to more complex driving environments.

3.2. Stability Analysis. To prove asymptotic stability of the closed-loop dynamics driven by the control action, we first define position, velocity, and acceleration errors with respect to the reference signals $x_{0}(t), v_{0}(t)$, and $a_{0}(t), i=1, \cdots, N$, as

$$
\begin{aligned}
& \bar{x}_{i}(t)=\left(x_{i}(t)-x_{0}(t)+i \cdot s\right), \\
& \bar{v}_{i}(t)=\left(v_{i}(t)-v_{0}(t)\right), \\
& \bar{a}_{i}(t)=\left(a_{i}(t)-a_{0}(t)\right) .
\end{aligned}
$$

We assume that the variation of the vehicle's velocity during the delay time can be ignored and the leader's Jerk $\dot{a}_{0}(t)$ is approximately zero. Based on the assumptions, we have $v_{j}(t-$ $\left.\tau_{j}\right) \approx v_{j}(t)$ and $\dot{a}_{0}(t)=0$. Then, we can rewrite the coupling control action $u_{i}(t)$ in terms of the state errors $\bar{x}_{i}(t), \bar{v}_{i}(t)$, and $\bar{a}_{i}(t)$. After performing some algebraic manipulation, we obtain the closed-loop dynamics for the generic $i$ th platoon vehicle:

$$
\begin{aligned}
\dot{\bar{x}}_{i}(t) & =\bar{v}_{i}(t), \\
\dot{\bar{v}}_{i}(t) & =\bar{a}_{i}(t), \\
\dot{\bar{a}}_{i}(t) & =\dot{a}_{i}(t)-\dot{a}_{0}(t) \\
= & \frac{1}{T_{i}}\left\{\sum_{j=1}^{N} \alpha_{i j} \beta_{1}\left[\bar{x}_{j}\left(t-\tau_{j}\right)-\bar{x}_{i}(t)\right]\right. \\
+ & \sum_{j=1}^{N} \alpha_{i j} \beta_{2}\left[\bar{v}_{j}\left(t-\tau_{j}\right)-\bar{v}_{i}(t)\right] \\
- & \left.b_{i}\left[\beta_{1} \bar{x}_{i}(t)+\beta_{2} \bar{v}_{i}(t)\right]-\left(1+b_{i} \beta_{3}\right) \bar{a}_{i}(t)\right\} .
\end{aligned}
$$


By defining $\bar{x}=\left[\bar{x}_{0}, \cdots, \bar{x}_{N}\right]^{T}, \bar{v}=\left[\bar{v}_{0}, \cdots, \bar{v}_{N}\right]^{T}, \bar{a}=\left[\bar{a}_{0}\right.$, $\left.\cdots, \bar{a}_{N}\right]^{T}$, and $\varepsilon=\left[\begin{array}{lll}\bar{x}^{T} & \bar{v}^{T} & \bar{a}^{T}\end{array}\right]^{T}$, the closed-loop dynamics of the platoon can be written in a more compact form:

$$
\dot{\varepsilon}(t)=Y_{0} \varepsilon(t)+\sum_{j=1}^{N} Y_{j} \varepsilon\left(t-\tau_{j}\right) .
$$

Here

$$
\begin{aligned}
& Y_{0} \\
& =\left[\begin{array}{ccc}
0_{N \times N} & I_{N \times N} & 0_{N \times N} \\
0_{N \times N} & 0_{N \times N} & I_{N \times N} \\
-\beta_{1} T(D+B) & -\beta_{2} T(D+B) & -\left(I+\beta_{3} B\right) T
\end{array}\right],
\end{aligned}
$$

and

$$
Y_{j}=\left[\begin{array}{ccc}
0_{N \times N} & 0_{N \times N} & 0_{N \times N} \\
0_{N \times N} & 0_{N \times N} & 0_{N \times N} \\
\beta_{1} T A_{j} & \beta_{2} T A_{j} & 0_{N \times N}
\end{array}\right],
$$

where

$$
\begin{gathered}
A_{j}=\left[\begin{array}{ccccc}
0 & \cdots & \alpha_{1 j} & \cdots & 0 \\
\vdots & \vdots & \vdots & \vdots & \vdots \\
0 & \cdots & \alpha_{j j} & \cdots & 0 \\
\vdots & \vdots & \vdots & \vdots & \vdots \\
0 & \cdots & \alpha_{N j} & \cdots & \vdots
\end{array}\right] \\
\text { and } T=\operatorname{diag}\left\{\frac{1}{T_{1}}, \ldots, \frac{1}{T_{N}}\right\} \in R^{N \times N} .
\end{gathered}
$$

Applying the Leibniz-Newton formula leads to

$$
\begin{aligned}
\varepsilon\left(t-\tau_{j}\right) & =\varepsilon(t)-\int_{-\tau_{j}}^{0} \dot{\varepsilon}(t+s) d s \\
& =\varepsilon(t)-\sum_{i=0}^{N} Y_{i} \int_{-\tau_{j}}^{0} \varepsilon\left(t+s-\tau_{i}\right) d s,
\end{aligned}
$$

where $\tau_{0}=0$. Substituting (15) into (11), we can obtain

$$
\dot{\varepsilon}(t)=F \mathcal{\varepsilon}(t)-\sum_{j=1}^{N} \sum_{i=0}^{N} Y_{j} Y_{i} \int_{-\tau_{j}}^{0} \varepsilon\left(t+s-\tau_{i}\right) d s,
$$

where

$$
F=\sum_{i=0}^{N} Y_{i}=\left[\begin{array}{ccc}
0_{N \times N} & I_{N \times N} & 0_{N \times N} \\
0_{N \times N} & 0_{N \times N} & I_{N \times N} \\
-\beta_{1} T H & -\beta_{2} T H & -\left(I+\beta_{3} B\right) T
\end{array}\right],
$$

with

$$
H=(L+B)
$$

From (12) and (13), we have $Y_{j} Y_{i}=0$ when $j=1, \cdots, N$ and $i=1, \cdots, N$. Hence, (16) can be rewritten as

$$
\dot{\varepsilon}(t)=F \mathcal{\varepsilon}(t)-\sum_{j=1}^{N} Y_{j} Y_{0} \int_{-\tau_{j}}^{0} \varepsilon(t+s) d s .
$$

Lemma 4 (see [35]). The matrix $H$ is positive stable if and only if node 0 is globally reachable in $\bar{G}$.

Let $H_{T}$ be the matrix defined by $H_{T}=T H$. According to Lemma $4, H_{T}$ is also positive stable since $T>0$.

Lemma 5. Let the matrix $F$ be as given in (17) and $\beta_{2}(1+$ $\left.b_{i} \beta_{3}\right)>\beta_{1} T_{i} ; F$ is Hurwitz stable if and only if $H_{T}$ is a positive stable matrix and

$$
\begin{aligned}
& \operatorname{Re}\left(\mu_{i}\right)\left(\frac{1+b_{i} \beta_{3}}{T_{i}}\right)\left[\beta_{2}\left(\frac{1+b_{i} \beta_{3}}{T_{i}}\right)-\beta_{1}\right] \\
& -\left(\beta_{2} \operatorname{Im}\left(\mu_{i}\right)\right)^{2}>0, \\
& \beta_{1}\left(\operatorname{Re}\left(\mu_{i}\right)\right)^{3}\left[\beta_{2}\left(\frac{1+b_{i} \beta_{3}}{T_{i}}\right)-\beta_{1}\right]^{2}+\beta_{1} \beta_{2} \operatorname{Re}\left(\mu_{i}\right) \\
& \cdot\left(\operatorname{Im}\left(\mu_{i}\right)\right)^{2}\left(\frac{1+b_{i} \beta_{3}}{T_{i}}\right)\left[\beta_{2}\left(\frac{1+b_{i} \beta_{3}}{T_{i}}\right)-3 \beta_{1}\right] \\
& -\beta_{1}\left(\operatorname{Im}\left(\mu_{i}\right)\right)^{2}\left[\beta_{1}\left(\frac{1+b_{i} \beta_{3}}{T_{i}}\right)^{3}\right. \\
& \left.+\left(\beta_{2}\right)^{3}\left(\operatorname{Re}\left(\mu_{i}\right)\right)^{2}+\left(\beta_{2}\right)^{3}\left(\operatorname{Im}\left(\mu_{i}\right)\right)^{2}\right]>0,
\end{aligned}
$$

where $\mu_{i}$ is the ith eigenvalue of $H_{T}$.

Proof (Sufficiency). Let $\lambda$ be the eigenvalue of $F$; then

$$
\begin{aligned}
\operatorname{det} & \left(\lambda I_{3 N \times 3 N}-F\right) \\
= & \left|\begin{array}{lll}
\lambda I_{N \times N} & -I_{N \times N} & 0_{N \times N} \\
0_{N \times N} & \lambda I_{N \times N} & -I_{N \times N} \\
\beta_{1} H_{T} & \beta_{2} H_{T} & \lambda I_{N \times N}+\left(I+\beta_{3} B\right) T
\end{array}\right| \\
= & \operatorname{det}\left(\lambda^{3} I_{N \times N}+\lambda^{2}\left(I+\beta_{3} B\right) T+\lambda \beta_{2} H_{T}+\beta_{1} H_{T}\right) \\
= & \prod_{i}^{N}\left[\lambda^{3}+\left(\frac{1+b_{i} \beta_{3}}{T_{i}}\right) \lambda^{2}+\beta_{2} \mu_{i} \lambda+\beta_{1} \mu_{i}\right] \\
= & \prod_{i}^{N} R_{i}\left(\lambda, \mu_{i}\right) .
\end{aligned}
$$

Noting that $H_{T}$ is a positive stable, i.e., $\operatorname{Re}\left(\mu_{i}\right)>0$, and choosing the control gains $\beta_{1}>0, \beta_{2}>0$, and $\beta_{3}>0$ such 
that the conditions (20) and (21) are satisfied, we have that, for the $i$ th polynomial $R_{i}\left(\lambda, \mu_{i}\right)$,

$$
\begin{aligned}
D_{1 i} & =\frac{1+b_{i} \beta_{3}}{T_{i}}, \\
D_{2 i} & =\operatorname{Re}\left(\mu_{i}\right)\left(\frac{1+b_{i} \beta_{3}}{T_{i}}\right)\left[\beta_{2}\left(\frac{1+b_{i} \beta_{3}}{T_{i}}\right)-\beta_{1}\right] \\
& -\left(\beta_{2} \operatorname{Im}\left(\mu_{i}\right)\right)^{2}, \\
D_{3 i} & =\beta_{1}\left(\operatorname{Re}\left(\mu_{i}\right)\right)^{3}\left[\beta_{2}\left(\frac{1+b_{i} \beta_{3}}{T_{i}}\right)-\beta_{1}\right]^{2} \\
+ & \beta_{1} \beta_{2} \operatorname{Re}\left(\mu_{i}\right)\left(\operatorname{Im}\left(\mu_{i}\right)\right)^{2}\left(\frac{1+b_{i} \beta_{3}}{T_{i}}\right) \\
\cdot & {\left[\beta_{2}\left(\frac{1+b_{i} \beta_{3}}{T_{i}}\right)-3 \beta_{1}\right]-\beta_{1}\left(\operatorname{Im}\left(\mu_{i}\right)\right)^{2} } \\
\cdot & {\left[\beta_{1}\left(\frac{1+b_{i} \beta_{3}}{T_{i}}\right)^{3}+\left(\beta_{2}\right)^{3}\left(\operatorname{Re}\left(\mu_{i}\right)\right)^{2}\right.} \\
& \left.+\left(\beta_{2}\right)^{3}\left(\operatorname{Im}\left(\mu_{i}\right)\right)^{2}\right]
\end{aligned}
$$

are all positive. According to [36,37], the roots of $R_{i}\left(\lambda, \mu_{i}\right)$ lie in the open left half of the complex plane. Thus, $F$ is Hurwitz stable.

(Necessity). If $H_{T}$ is not positive stable, there exists $\operatorname{Re}\left(\mu_{i}\right)$ which is less than or equal to zero. Then, the corresponding $D_{2 i}$ will be less than or equal to zero. This contradicts the fact that the matrix $F$ is Hurwitz stable.

Theorem 6. Consider system (11) and take the control parameters $\beta_{1}, \beta_{2}$, and $\beta_{3}$ as in Lemma 5. Then, if and only if node 0 is globally reachable in $\bar{G}$, there exists a constant $\widehat{\tau}>0$, such that when $0 \leq \tau_{j}<\widehat{\tau}(j=1, \cdots, N)$, the consensus is reached asymptotically; i.e.,

$$
\lim _{t \longrightarrow \infty} \varepsilon(t)=0 .
$$

Proof (Sufficiency). Choose appropriate control parameters based on Lemma 5 . Since node 0 is globally reachable in $\bar{G}$, $H_{T}$ is a positive stable. According to Lemma 5, $F$ is Hurwitz stable. There exists a positive-definite matrix $P \in R^{3 N \times 3 N}$ to satisfy

$$
P F+F^{T} P=-I_{3 N \times 3 N} .
$$

Consider the following Razumikhin function for system (19):

$$
V(\varepsilon)=\varepsilon^{T} P \varepsilon,
$$

which satisfies

$$
\lambda_{\text {min }}(P)\|\varepsilon\|^{2} \leq V(\varepsilon) \leq \lambda_{\max }(P)\|\varepsilon\|^{2} .
$$

From (26), we have

$$
\begin{aligned}
\dot{V}(\varepsilon)= & \dot{\varepsilon}^{T} P \varepsilon+\varepsilon^{T} P \dot{\varepsilon} \\
= & \varepsilon^{T}\left(P F+F^{T} P\right) \varepsilon \\
& -2 \sum_{j=1}^{N} \sum_{i=0}^{N} \varepsilon^{T} P Y_{j} Y_{0} \int_{-\tau_{j}}^{0} \varepsilon(t+s) d s .
\end{aligned}
$$

According to Lemma 3, let $c=-Y_{j}^{T} Y_{0}^{T} P \varepsilon, d=\varepsilon(t+s)$, and $E=P^{-1}$; then (28) becomes

$$
\begin{aligned}
\dot{V}(\varepsilon) \leq & -\varepsilon^{T} \varepsilon+\sum_{j=1}^{N} \sum_{i=0}^{N} \tau_{j} \varepsilon^{T}\left(P Y_{j} Y_{0} P^{-1} Y_{0}{ }^{T} Y_{j}{ }^{T} P\right) \varepsilon \\
& +\sum_{j=1}^{N} \sum_{i=0}^{N} \int_{-\tau_{j}}^{0} \varepsilon^{T}(t+s) P \varepsilon(t+s) d s .
\end{aligned}
$$

Take $\varphi_{4}(s)=\xi$ s for some constant $\xi>1$. When

$$
V(\varepsilon(t+\theta))<\xi V(\varepsilon(t)), \quad-\tau \leq \theta \leq 0,
$$

we have

$$
\begin{aligned}
\dot{V}(\varepsilon) \leq & -\varepsilon^{T} \varepsilon \\
& +\sum_{j=1}^{N} \sum_{i=0}^{N} \tau_{j} \varepsilon^{T}\left(P Y_{j} Y_{0} P^{-1} Y_{0}^{T} Y_{j}^{T} P+\xi P\right) \varepsilon,
\end{aligned}
$$

so, if

$$
\tau_{j}<\widehat{\tau}=\frac{1}{\left\|P Y_{j} Y_{0} P^{-1} Y_{0}{ }^{T} Y_{j}^{T} P+\xi P\right\|},
$$

then $\dot{V}(\varepsilon) \leq-\eta \varepsilon^{T} \varepsilon$ for some constant $\eta>0$. Thus, the conclusion follows from Theorem 1 .

(Necessity). Notice that system (11) is asymptotically stable for any $\tau_{j}<\widehat{\tau}, j=1, \cdots, N$. For the special case $\tau_{j}=0$, from (19) the system $\dot{\varepsilon}(t)=F \mathcal{\varepsilon}(t)$ is asymptotically stable. The eigenvalues of $F$ have negative real-parts, which implies that $H$ is positive stable. According to Lemma 4, node 0 is globally reachable in $\bar{G}$.

\section{Simulations}

4.1. Simulation Setting. We adopt PLEXE simulator [38] in our simulation. PLEXE integrates the network simulator OMNeT++/MiXiM and the road traffic simulator SUMO, which are used to simulate $\mathrm{V} 2 \mathrm{~V}$ communication based on the 802.11p standard and the vehicle dynamics with the consensus algorithm, respectively. The parameters for the traffic simulation and consensus control algorithm are specified in Table 1. Control parameters are selected to guarantee consensus according to Theorem 6 . The parameters of delay are not set since they are implemented in PLEXE to simulate more realistic vehicle dynamics.

We select a typical communication topology: the leaderand predecessor-following topology considering information from both the preceding vehicle and the leader (see Figure 1). 
TABLE 1: Parameters for the traffic simulation and consensus control algorithm.

\begin{tabular}{lccc}
\hline Parameter & Value & Parameter & Value \\
\hline Vehicle length $l_{i}$ & $4 \mathrm{~m}$ & Maximum acceleration & $3 \mathrm{~m}^{2} / \mathrm{s}$ \\
Stable speed & $25 \mathrm{~m} / \mathrm{s}$ & Maximum deceleration & $5 \mathrm{~m}^{2} / \mathrm{s}$ \\
Vehicle drivetrain $T_{i}$ & $0.5 \mathrm{~s}$ & Maximum velocity & $35 \mathrm{~m} / \mathrm{s}$ \\
Control parameters & $\beta_{1}=2, \beta_{2}=2, \beta_{3}=3$ & Standstill distance $s$ & $15 \mathrm{~m}$ \\
if $b_{i}>0$ & $b_{i}=10$ & & \\
\hline
\end{tabular}

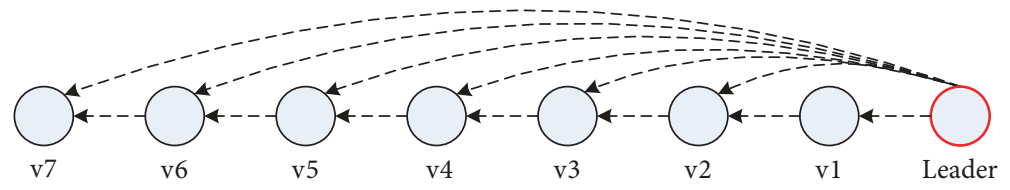

FIgURE 1: The information flow topology used in simulations: leader- and predecessor-following topology.
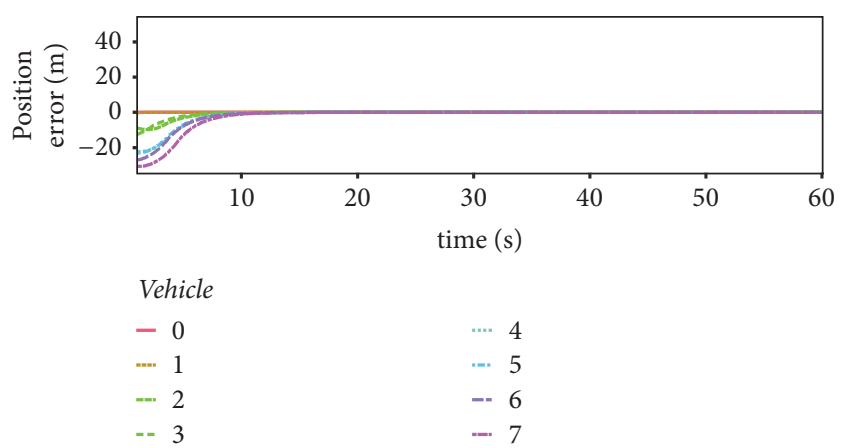

(a)
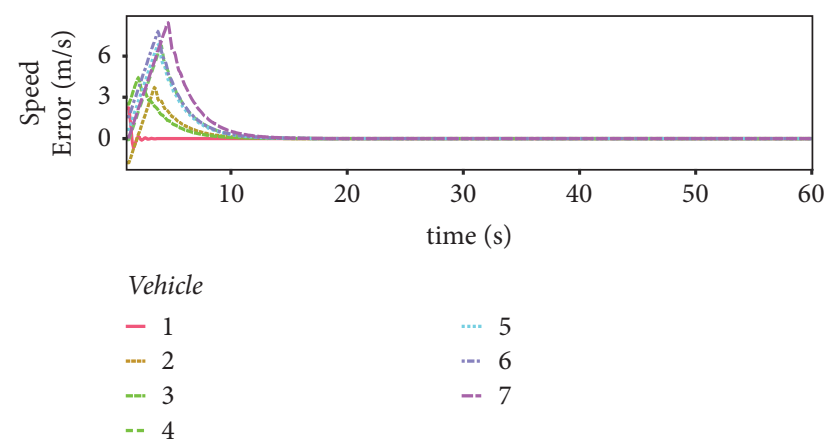

(b)

FIGURE 2: Platoon performance for the initial scenario: (a) the position error $x_{i}(t)-x_{0}(t)+i \cdot s$; (b) the speed error $v_{i}(t)-v_{0}(t)$.

4.2. Platoon Formation and Maintenance. We first consider the platoon composed of seven following vehicles and a leader initially starting from different positions with different speeds. It is assumed that there are no packet losses to avoid the effect of the communication on the system performance. Figure 2 shows the results for this initial scenario. The results illustrate that all vehicles converge toward the desired positions satisfying the spacing policy requirements (see Figure 2(a)) and reach the leader speed (see Figure 2(b)). The platoon forms and maintains the behavior imposed by the leader. The results confirm the ability of the proposed consensus approach to create and maintain the platoon.

4.3. Performance for Perturbations. We consider a single large perturbation scenario similarly reported in [24], where the leader decelerates from $25 \mathrm{~m} / \mathrm{s}$ to $10 \mathrm{~m} / \mathrm{s}$, then keeps the speed at $10 \mathrm{~m} / \mathrm{s}$ for some time, and accelerates with $2 \mathrm{~m}^{2} / \mathrm{s}$ from $10 \mathrm{~m} / \mathrm{s}$ to $25 \mathrm{~m} / \mathrm{s}$. The test is used to evaluate the ability of the approach in tracking the leader motion. The simulation results are shown in Figure 3. We can see that all vehicles almost simultaneously start to decelerate/accelerate (see Figure 3(c)) and the following vehicles can be fast and correctly track the leader speed (see Figure 3(b)), which confirms the tracking performance of the proposed algorithm.

To further verify the efficiency of the presented algorithm, we consider a periodic disturbance, where the following sinusoidal disturbance is added onto the leading vehicle speed:

$$
\delta(t)=A \sin (0.2 \pi t), \quad A=2.7 \mathrm{~m} / \mathrm{s} .
$$

The goal is to investigate if the errors are amplified along the vehicle string (string stability). Figure 4 illustrates the simulation results. We can see that the relative position (see Figure 4(a)) and relative speed (see Figure 4(b)) are attenuated along the platoon, which illustrates the proposed approach can counteract the influence of the periodic disturbance and maintain the string stability.

We next study another kind of perturbation coming from security risks discussed by [16]. We consider one of the attacks, i.e., spoofing, and adopt similar scenario presented in [16]. For the initial scenario in Section 4.2, an internal adversary controls the third vehicle and injects fraudulent information by means of setting its acceleration to the maximum value at $t=30 \mathrm{~s}$. This control lasts for two seconds. The simulation results are shown in Figure 5. It can be seen that after about $10 \mathrm{~s}$ transient stage, the members' states could be recovered. If we extend the voting technique developed in [16] to our algorithm, it will be possible to obtain better results, which will be left in our future work. 

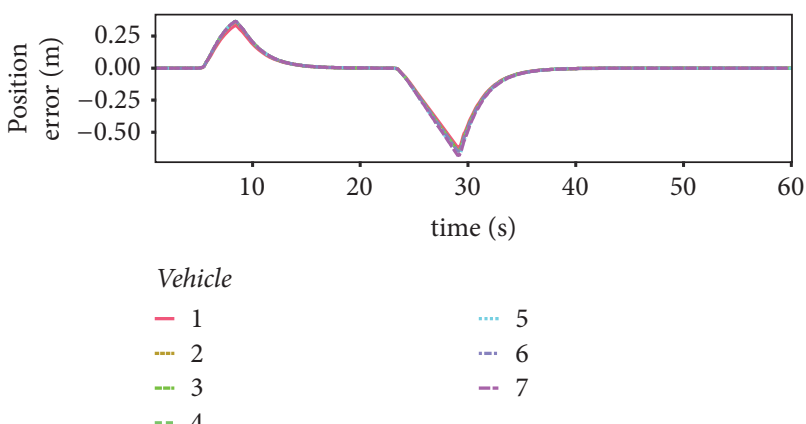

(a)
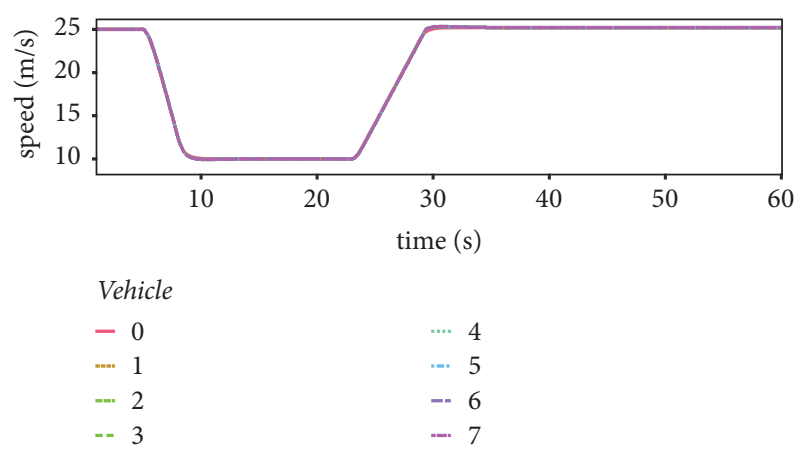

(b)
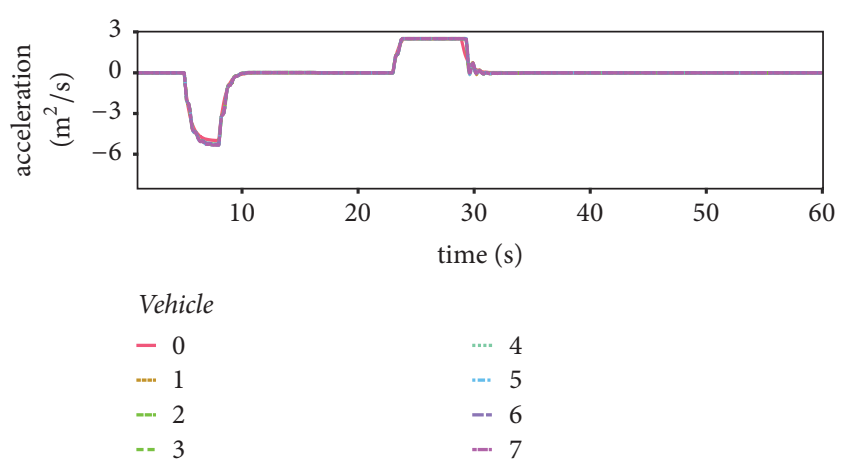

(c)

FIGURE 3: Platoon performance for a single large perturbation scenario: (a) the position error $x_{i}(t)-x_{0}(t)+i \cdot s$; (b) speed; (c) acceleration.

4.4. Impact of Platoon Length. In this subsection, we study the performance of the proposed consensus algorithm for different platoon lengths. We consider the platoons with 4 , $7,10,13$, and 16 vehicles. Figure 6 shows the errors of the last vehicle of the platoon with respect to the leader 0 under different platoon lengths for the initial scenario. We can see that the platoons reach the consensus for different platoon lengths. The longer the platoon length is, the longer the convergence time is. From Figure 6, it also can be seen that the longer the platoon length is, the larger the speed error is. This is because the last vehicle of the platoon minimizes the larger position error with respect to the leader.

Figure 7 illustrates the state errors of the last vehicle of the platoon with respect to the leader under different platoon lengths for the sinusoidal disturbance scenario. From
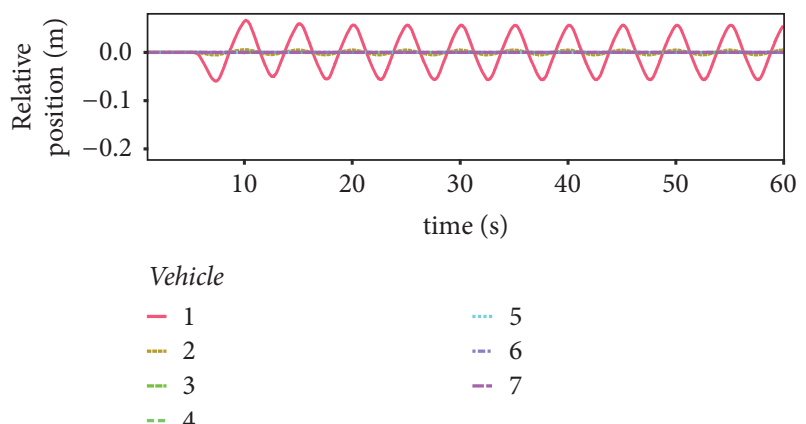

(a)
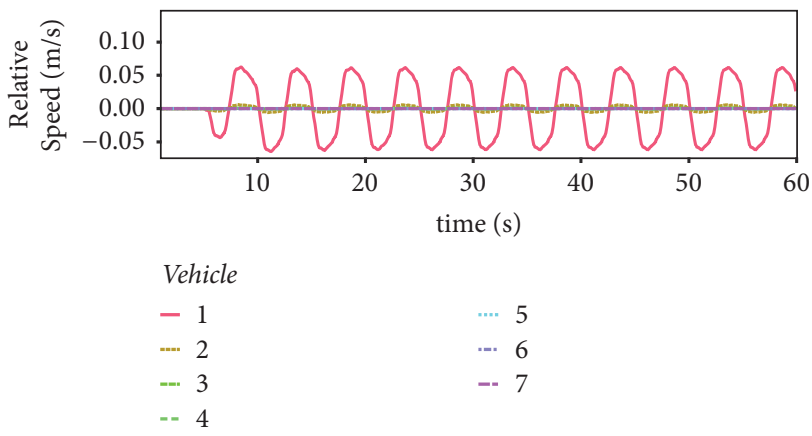

(b)
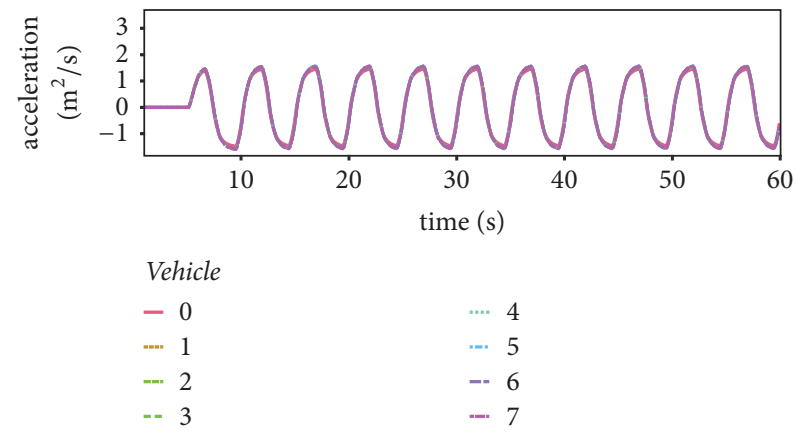

(c)

FIGURE 4: Platoon performance for the sinusoidal disturbance scenario: (a) the relative position $x_{i-1}(t)-x_{i}(t)+s$; (b) the relative speed $v_{i-1}(t)-v_{i}(t) ;(\mathrm{c})$ acceleration.

Figure 7, we can see that the strategy is able to track the leader's motion for different platoon lengths.

\section{Conclusions}

In this paper, we have proposed a novel third-order consensus strategy for the vehicle platoon and have proven the asymptotically stability of the platooning algorithm in presence of time-varying delays. We have tested several traffic scenarios including the initial case, the large perturbation, the sinusoidal disturbance, and the perturbation coming from security risks. The simulation results illustrate the effectiveness of the approach and confirm the robustness of the proposed strategy in the presence of perturbations. In addition, the proposed consensus control approach shows the effectiveness for different platoon lengths. Future work will 


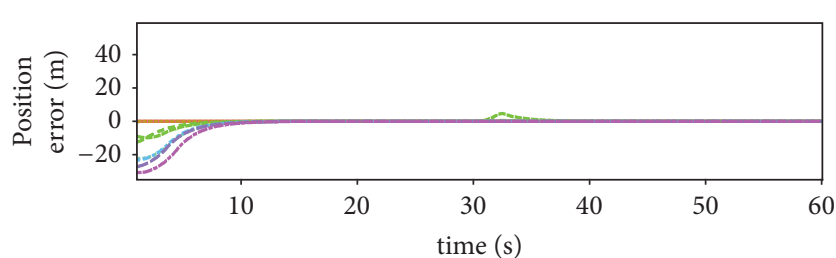

Vehicle

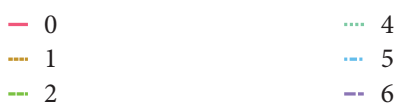

$\begin{array}{ll}- & - \\ - & \end{array}$

(a)

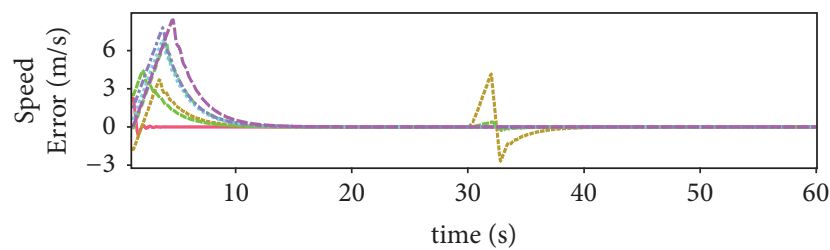

Vehicle

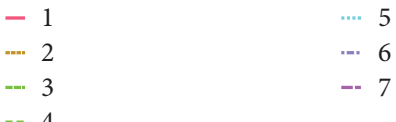

(b)

FIGURE 5: Platoon performance for the initial scenario under spoofing attack: (a) the position error $x_{i}(t)-x_{0}(t)+i \cdot s$; (b) the speed error $v_{i}(t)-v_{0}(t)$.

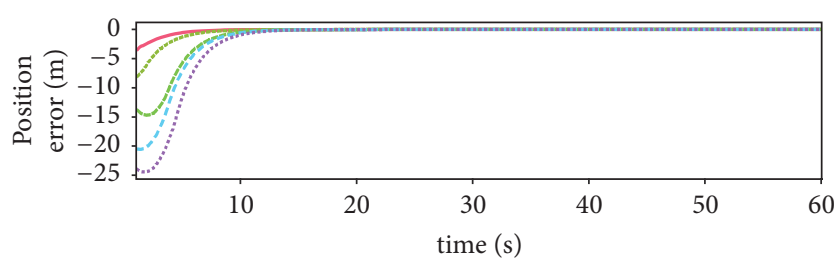

Vehicle Number

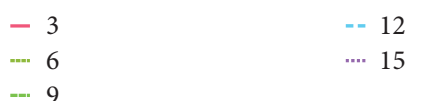

(a)

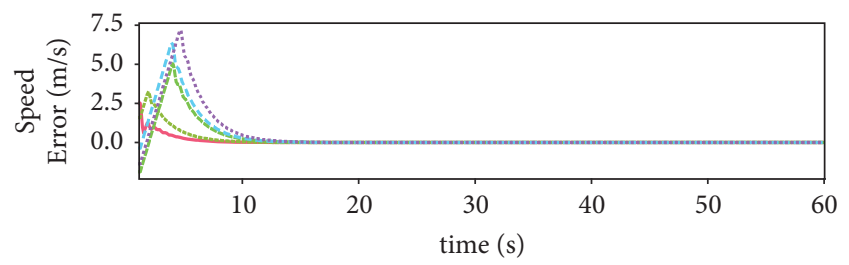

Vehicle Number

$\begin{array}{rr}-3 & --12 \\ --.-6 & 6 \\ -- & 9\end{array}$

(b)

FIGURE 6: Platoon performance in the presence of the initial scenario under different platoon lengths: (a) the position error $x_{i}(t)-x_{0}(t)+$ $i \cdot s$; (b) the speed error $v_{i}(t)-v_{0}(t)$.

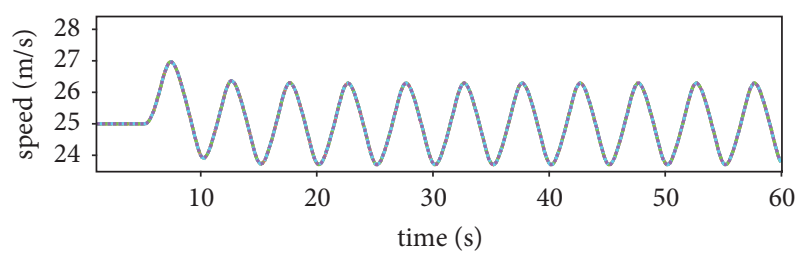

Vehicle Number

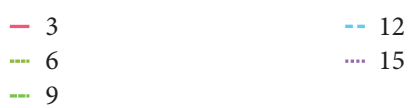

(a)

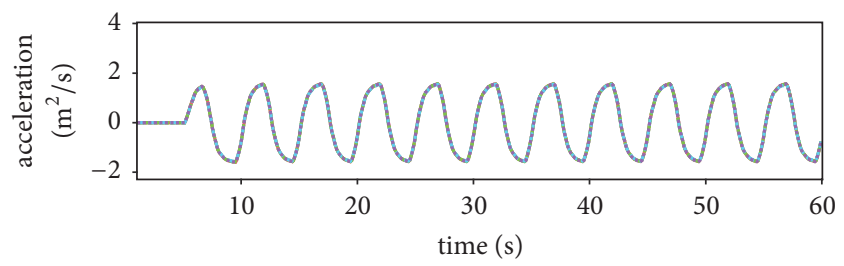

Vehicle Number

$\begin{array}{rr}-3 & -12 \\ -\ldots-2 & 6 \\ --- & 9\end{array}$

(b)

FIGURE 7: Platoon performance in the presence of the sinusoidal disturbance scenario under different platoon lengths: (a) speed; (b) acceleration.

be devoted to investigating the effect of switching communication network topologies. Furthermore, more sophisticated spacing strategy should be introduced to determine the desired distance of vehicle. In addition, how to choose the optimal control parameter values will be studied in future work.

\section{Data Availability}

The data used to support the findings of this study are available from the corresponding author upon request.

\section{Conflicts of Interest}

The authors declare that they have no conflicts of interest.

\section{Acknowledgments}

This work was supported by the National Natural Science Foundation of China (no. 11772264).

\section{References}

[1] J. Axelsson, "Safety in vehicle platooning: A systematic literature review," IEEE Transactions on Intelligent Transportation Systems, vol. 18, no. 5, pp. 1033-1045, 2017.

[2] P. Kavathekar and Y. Chen, "Vehicle platooning: A brief survey and categorization," in Proceedings of the ASME 2011 International Design Engineering Technical Conferences and Computers 
and Information in Engineering Conference, IDETC/CIE 2011, pp. 829-845, Washington, DC, USA, August 2011.

[3] L. Xu, L. Y. Wang, G. Yin, and H. Zhang, "Communication information structures and contents for enhanced safety of highway vehicle platoons," IEEE Transactions on Vehicular Technology, vol. 63, no. 9, pp. 4206-4220, 2014.

[4] S. E. Shladover, C. A. Desoer, J. K. Hedrick et al., "Automated vehicle control developments in the PATH program," IEEE Transactions on Vehicular Technology, vol. 40, no. 1, pp. 114-130, 1991.

[5] Y. Zheng, S. E. Li, K. Li, F. Borrelli, and J. K. Hedrick, "Distributed model predictive control for heterogeneous vehicle platoons under unidirectional topologies," IEEE Transactions on Control Systems Technology, vol. 25, no. 3, pp. 899-910, 2017.

[6] F. Lin, M. Fardad, and M. R. Jovanovic, "Optimal control of vehicular formations with nearest neighbor interactions," IEEE Transactions on Automatic Control, vol. 57, no. 9, pp. 2203-2218, 2012.

[7] X. Guo, J. Wang, F. Liao, and R. S. H. Teo, "Distributed adaptive integrated-sliding-mode controller synthesis for string stability of vehicle platoons," IEEE Transactions on Intelligent Transportation Systems, vol. 17, no. 9, pp. 2419-2429, 2016.

[8] J. Ploeg, D. P. Shukla, N. Van De Wouw, and H. Nijmeijer, "Controller synthesis for string stability of vehicle platoons," IEEE Transactions on Intelligent Transportation Systems, vol. 15, no. 2, pp. 854-865, 2014.

[9] J. A. Fax and R. M. Murray, "Information flow and cooperative control of vehicle formations," IEEE Transactions on Automatic Control, vol. 49, no. 9, pp. 1465-1476, 2004.

[10] W. Ren, "Consensus strategies for cooperative control of vehicle formations," IET Control Theory \& Applications, vol. 1, no. 2, pp. 505-512, 2007.

[11] L. Y. Wang, A. Syed, G. G. Yin, A. Pandya, and H. Zhang, "Control of vehicle platoons for highway safety and efficient utility: consensus with communications and vehicle dynamics," Journal of Systems Science and Complexity, vol. 27, no. 4, pp. 605-631, 2014.

[12] M. di Bernardo, A. Salvi, and S. Santini, "Distributed consensus strategy for platooning of vehicles in the presence of timevarying heterogeneous communication delays," IEEE Transactions on Intelligent Transportation Systems, vol. 16, no. 1, pp. 102112, 2015.

[13] M. di Bernardo, P. Falcone, A. Salvi, and S. Santini, "Design, analysis, and experimental validation of a distributed protocol for platooning in the presence of time-varying heterogeneous delays," IEEE Transactions on Control Systems Technology, vol. 24, no. 2, pp. 413-427, 2016.

[14] S. Santini, A. Salvi, A. S. Valente, A. Pescapè, M. Segata, and R. Lo Cigno, "A consensus-based approach for platooning with inter-vehicular communications," in Proceedings of the 34th IEEE Annual Conference on Computer Communications and Networks (INFOCOM '15), pp. 1158-1166, May 2015.

[15] S. Santini, A. Salvi, A. S. Valente, A. Pescapé, M. Segata, and R. Lo Cigno, "A consensus-based approach for platooning with intervehicular communications and its validation in realistic scenarios," IEEE Transactions on Vehicular Technology, vol. 66, no. 3, pp. 1985-1999, 2017.

[16] A. Petrillo, A. Pescapé, and S. Santini, "A collaborative approach for improving the security of vehicular scenarios: The case of platooning," Computer Communications, vol. 122, pp. 59-75, 2018.
[17] M. Yan, Y. Tang, P. Yang, and L. Zuo, "Consensus based platoon algorithm for velocity-measurement-absent vehicles with actuator saturation," Journal of Advanced Transportation, vol. 2017, Article ID 8023018, 2017.

[18] A. Salvi, S. Santini, and A. S. Valente, "Design, analysis and performance evaluation of a third order distributed protocol for platooning in the presence of time-varying delays and switching topologies," Transportation Research Part C: Emerging Technologies, vol. 80, pp. 360-383, 2017.

[19] M. di Bernardo, A. Salvi, S. Santini, and A. S. Valente, "Thirdorder consensus in vehicles platoon with heterogeneous timevarying delays," IFAC-PapersOnLine, vol. 48, no. 12, pp. 358$363,2015$.

[20] M. Saeednia and M. Menendez, "A consensus-based algorithm for truck platooning," IEEE Transactions on Intelligent Transportation Systems, vol. 18, no. 2, pp. 404-415, 2017.

[21] Z. Wang, G. Wu, and M. J. Barth, "Developing a distributed consensus-based cooperative adaptive cruise control system for heterogeneous vehicles with predecessor following topology," Journal of Advanced Transportation, vol. 2017, Article ID 1023654, 2017.

[22] J. C. Zegers, E. Semsar-Kazerooni, J. Ploeg, N. van de Wouw, and H. Nijmeijer, "Consensus control for vehicular platooning with velocity constraints," IEEE Transactions on Control Systems Technology, no. 99, pp. 1-14, 2017.

[23] D. Jia and D. Ngoduy, "Platoon based cooperative driving model with consideration of realistic inter-vehicle communication," Transportation Research Part C: Emerging Technologies, vol. 68, pp. 245-264, 2016.

[24] D. Jia and D. Ngoduy, "Enhanced cooperative car-following traffic model with the combination of V2V and V2I communication," Transportation Research Part B: Methodological, vol. 90, pp. 172-191, 2016.

[25] D. Jia, D. Ngoduy, and H. L. Vu, "A multiclass microscopic model for heterogeneous platoon with vehicle-to-vehicle communication," Transportmetrica B: Transport Dynamics, pp. 1-25, 2018.

[26] Z. Li and Y. Liu, "Analysis of stability and density waves of traffic flow model in an ITS environment," The European Physical Journal B-Condensed Matter and Complex Systems, vol. 53, no. 3, pp. 367-374, 2006.

[27] D. F. Xie, Z. Y. Gao, and X. M. Zhao, "Stabilization of traffic flow based on the multiple information of preceding cars," Computer Physics Communications, vol. 3, no. 4, pp. 899-912, 2008.

[28] Y. Li, D. Sun, W. Liu et al., "Modeling and simulation for microscopic traffic flow based on multiple headway, velocity and acceleration difference," Nonlinear Dynamics, vol. 66, no. 1-2, pp. 15-28, 2011.

[29] Y. M. Hu, T. S. Ma, and J. Z. Chen, "An extended multianticipative delay model of traffic flow," Communications in Nonlinear Science and Numerical Simulation, vol. 19, no. 9, pp. 3128-3135, 2014.

[30] R. A. Horn and C. R. Johnson, Matrix Analysis, Cambridge University Press, 1990.

[31] J. K. Hale and S. M. Verduyn Lunel, Introduction to FunctionalDifferential Equations, Springer Science \& Business Media, 2013.

[32] J. Hadamard, "Étude sur les propriétés des fonctions entières et en particulier d'une fonction considerée par Riemann," Journal De Mathématiques Pures Et Appliquées, vol. 58, pp. 171-215, 1893.

[33] R. Rajamani, Vehicle Dynamics and Control, Springer, Berlin, Germany, 2012. 
[34] D. Swaroop, J. K. Hedrick, C. C. Chien, and P. Ioannou, "A Comparision of Spacing and Headway Control Laws for Automatically Controlled Vehicles," Vehicle System Dynamics, vol. 23, no. 1, pp. 597-625, 1994.

[35] J. Hu and Y. Hong, "Leader-following coordination of multiagent systems with coupling time delays," Physica A: Statistical Mechanics and its Applications, vol. 374, no. 2, pp. 853-863, 2007.

[36] C. Parks and V. Hahn, Stability Theory, Prentice Hall, Upper Saddle River, NJ, USA, 1993.

[37] E. Frank, "On the zeros of polynomials with complex coefficients," Bulletin of the American Mathematical Society, vol. 52, no. 2, pp. 144-157, 1946.

[38] M. Segata, S. Joerer, B. Bloessl, C. Sommer, F. Dressler, and R. L. Cigno, "Plexe: A platooning extension for Veins," in Proceedings of the 2014 IEEE Vehicular Networking Conference, VNC 2014, pp. 53-60, Paderborn, Germany, December 2014. 


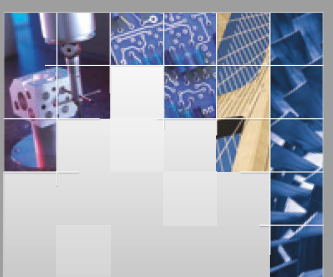

\section{Enfincering}
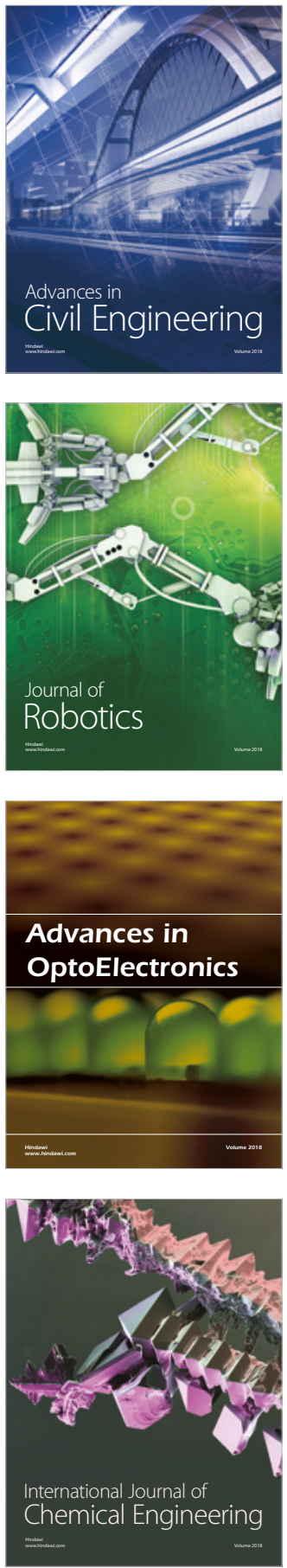

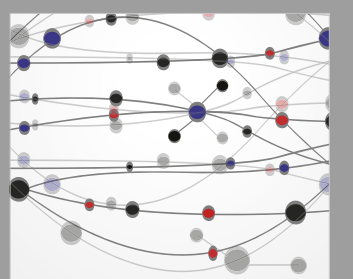

\section{Rotating \\ Machinery}

The Scientific World Journal

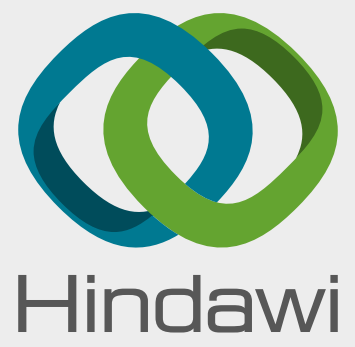

Submit your manuscripts at

www.hindawi.com
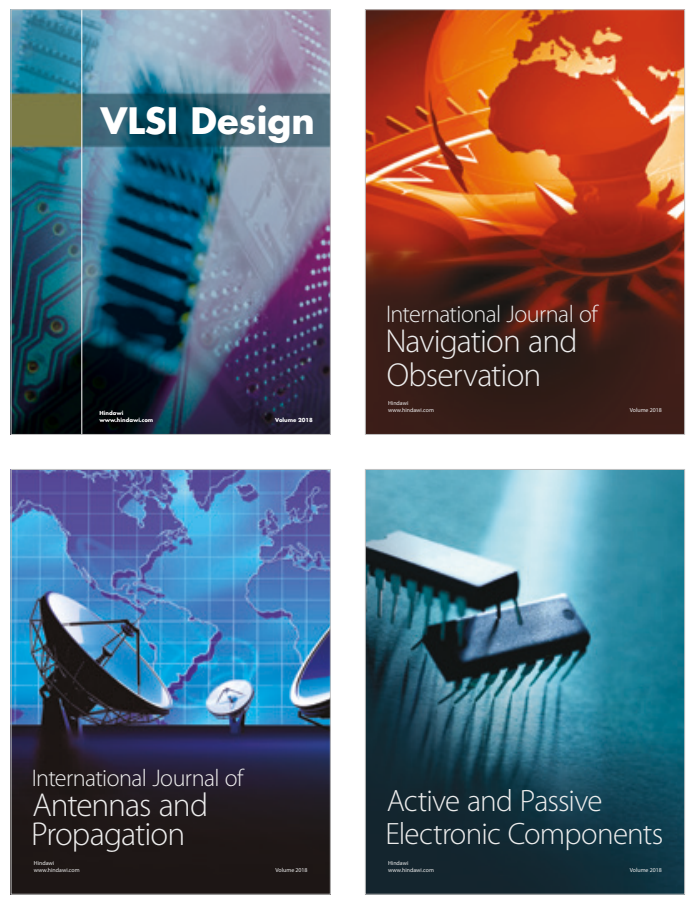
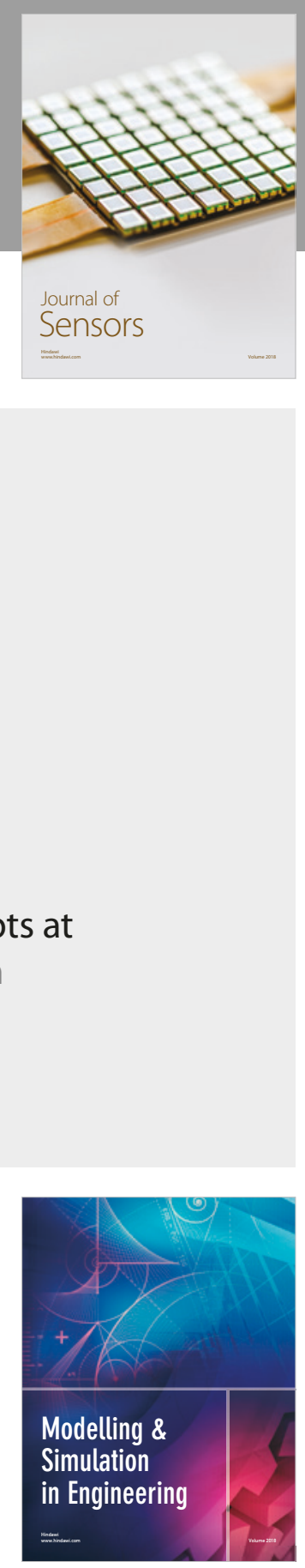

\section{Advances \\ Multimedia}
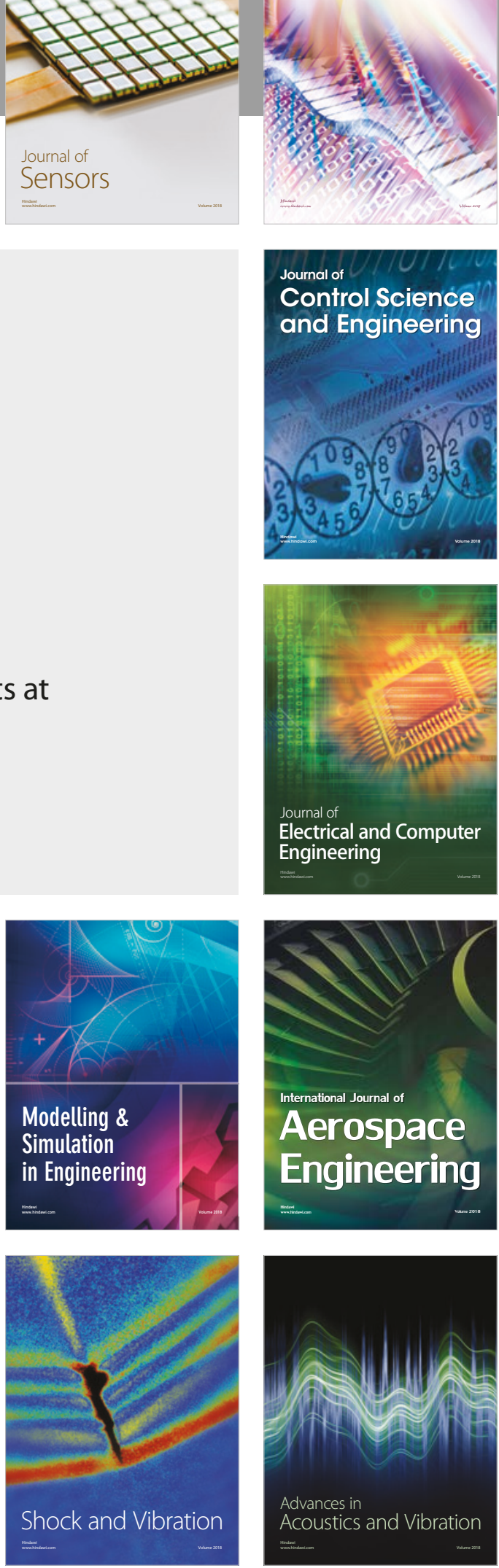